\title{
Matching patient and physician preferences in designing a primary care facility network
}

\author{
ED Güneş ${ }^{1}$, H Yaman ${ }^{2}$, B Çekyay ${ }^{3}$ and V Verter ${ }^{4 *}$ \\ ${ }^{1}$ Koç University, Istanbul, Turkey; ${ }^{2}$ Bilkent University, Ankara, Turkey; ${ }^{3}$ Doğuş University, Istanbul, \\ Turkey; and ${ }^{4}$ McGill University, Quebec, Canada
}

This paper introduces an integer programming model for planning primary care facility networks, which accounts for the interests of different stakeholders while maximizing access to health care. Physician allocation to health-care facilities is explicitly modelled, which allows consideration of physician incentives in the planning phase. An illustrative case study in the Turkish primary care system is presented to show the implications of focusing on patient or physician preferences in the planning phase. A discussion of trade-offs between the different stakeholder preferences and some recommendations for modelling choices to match these preferences are provided. In the context of this case, we found that using an access measure that decays with distance, and incorporating nearest allocation constraints improves performance for all stakeholders. We also show that increasing the number of physicians may have adverse affects on access measures when physician preferences are addressed.

Journal of the Operational Research Society (2014) 65, 483-496. doi:10.1057/jors.2012.71

Published online 4 July 2012

Keywords: public facility location; physician allocation; physician preference; access; Turkey

\section{Introduction}

Primary health care is the first level of contact in a healthcare system. Its significance is recognized by the 1978 Alma-Ata declaration of the World Health Organization, and since then developing a strong primary health-care system has been a prime goal for health policymakers (WHO, 1978). Starfield (1998) defines primary care as 'that level of a health service system that provides entry into the system, provides person focused care over time, provides care for all but very uncommon or unusual conditions, and coordinates or integrates care provided elsewhere or by others'.

One important dimension of performance for the primary health-care system is access to services (Macinko et al, 2003), defined as the ease with which the health-care services are obtained. An important component of access is 'geographical access', which is measured by the distance to the primary care practice. Improving access has been a major concern in health services planning, and is a topic investigated by a vast literature on public facility location (see Daskin and Dean, 2005 for a review).

One major shortcoming of the existing literature on health-care facility location is the outright emphasis on the patients' access to facilities, while ignoring the other

\footnotetext{
${ }^{*}$ Correspondence: $V$ Verter, Desautels Faculty of Management, McGill University, 1001 Sherbrooke St. W., Montreal, Quebec H3A 1G5, Canada.

E-mail: vedat.verter@mcgill.ca
}

stakeholder preferences. Health facility location problem is different from traditional facility location problem, as service provision is possible only in presence of physicians, an important stakeholder of health services. Indeed, ensuring availability of physicians in rural and under-served areas has been a major concern for health policymakers. For example, Canada is experiencing a shortage of General Practitioners (GPs), with an insufficient supply of physicians, and a decreasing ratio of physician to population ratios in rural regions (Pong and Pitblado, 2005; Gladu, 2007; Rourke, 2008). The percentage of medical school students in Canada, selecting family medicine as their first choice fell from $40 \%$ in 1980 to $24 \%$ in 2003 (MacKean and Gutkin, 2003). In the US, there is an increasing demand induced by improved health-care coverage by the health system reforms, while American Medical Colleges' Center for Workforce Studies estimates a shortage of 46000 primary care doctors by 2025 (O'Reilly, 2010). Massachusetts Medical Society reports family medicine as one of the specialties with severe labour market conditions (Massachusetts Medical Society, 2007). Note also that there is an inequitable distribution of the available supply of physician workforce as most physicians prefer to work in central urban areas and rural areas face more risk of a physician shortage (Kindig and Movassaghi, 1989; Gladu 2007).

This situation of supply challenges in primary care services calls for a certain level of central planning. Primary health-care facility planning models should account for the 
physician incentives so as to increase the attractiveness of facilities for physicians and decrease the risk of falling short of physician supply for the planned facilities. This paper is concerned with the problem of planning for primary health-care facilities to improve access while taking into consideration the preferences of the physicians and patients. The main contribution is in the explicit modelling of physician allocation decisions in a facility location model, which allows searching for a solution that would be more attractive for physicians, by an appropriate choice of model parameters.

The primary health system considered in this paper is a family practice system, that is, each physician is responsible for a patient list (also called 'the patient panel'). This is a widely applied system in Europe, Canada, as well as in the US, and the problem studied in this paper is relevant for such contexts. For example in the UK, a strong family practice tradition exists, where GPs establish practices. Most practices operate a geographical proximity-based 'catchment area' system, where only those within the area are able to register.

In such a context, organizing for primary care involves the problem of determining the location and catchment areas of GP practices. One realistic instance of this problem has been encountered in Turkey. The planning problem described here has been the mission of the provincial health officers in Turkey during the health system reform in the years 2005-2010, establishing the family practice system. The decisions involved choosing locations of clinics from among existing alternative locations, and assigning the number of family doctor posts to those clinics so as to maximize access of the population to family health centres (FHCs). The transformation period has been challenging because of a resistance from the Association of Physicians. And therefore, it has been particularly important to address physicians' concerns in the facility planning phase.

The present paper explicitly models physician allocation to primary health-care facilities. This facilitates accounting for the physicians during the facility planning phase. To our knowledge, this is the first paper that addresses physician preferences in location-allocation planning of health-care systems.

We develop a discrete location-allocation model for regional planning of primary health-care services to address three sets of decisions to maximize access: (1) location of FHCs; (2) the number of physicians at each health centre; (3) allocation of population centres to FHCs. This model takes the perspective of a central planner and provides an analytical framework to analyse the trade-offs among the preferences of the two stakeholders of healthcare systems: patients and physicians. The objective of the central planner is to maximize access, represented by two alternative objective functions: total coverage and expected participation. We then evaluate the performance of the solutions of alternative models based on other criteria representing preferences of patients and physicians. The preferences of these stakeholders are represented by a focus on different performance measures for which the solution can be evaluated.

The proposed model provides an analytical framework to gain a solid understanding of the nature of these tradeoffs. The implementation of our model using data from Turkey shows that for most instances, the following modelling choices ensure a relatively good match of patient and physician preferences: (1) using a participation function to measure access as opposed to a coverage objective, (2) enforcing allocation of patients to the nearest open facility, (3) enforcing a lower bound on the number of physicians assigned to a facility. Moreover, we find that when physician preferences are addressed, increasing the number of physicians may have adverse effects on access and average travel distance.

The rest of the paper is organized as follows. Section 2 briefly reviews the literature, and Section 3 introduces the problem. Section 4 presents our model formulation. In Section 5, a case study based on data obtained from a province in Turkey is presented and several numerical experiments are discussed. We conclude in Section 6.

\section{Literature review}

In this review we briefly discuss recent papers studying the problem of locating health-care facilities. For more information on general facility location problems including dynamic and probabilistic location problems, we refer the interested readers to Owen and Daskin (1998) and Smith et al (2009).

A majority of health-care facility location models are extensions of three standard facility location models: the maximal covering model, $p$-median model, and $p$-centre model. Although there is a vast literature on this type of problems (for reviews see Rahman and Smith, 1999; Daskin and Dean, 2005), the health-care facility location is still a very active research area (Rais and Viana, 2011).

The maximal coverage location problem, first defined by Church and ReVelle (1974), maximizes population covered for a fixed number of facilities. Berman and Krass (2002) introduce the generalized maximal coverage location problem where different levels of coverage exist, defined by a decreasing step function of the distance to the closest facility. Marianov and Taborga (2001) consider the problem of locating public health centres providing subsidized services to low-income patients. The model assumes that public health centres also provide paid services to highincome patients who are willing to be allocated to a public health centre if it is closer than any other private health centre. Jia et al (2007) analyse the problem of locating medical facilities that are primarily used in case of a largescale emergency such as earthquakes, terrorist attacks, etc. 
They propose a covering model, a $p$-median model, and a $p$-centre model for a large-scale emergency, characterized by sudden and enormous demand, and low frequency.

Allocating a patient to a health centre may not imply that the patient has unlimited access to health services. In particular, patients may be reluctant to participate in preventive programmes if the health centre is too far away. In this regard, the expected participation to the medical services could be a better proxy of the access in health service systems. Zhang et al (2009) model preventive health-care facility location problem to maximize participation. They define the participation probability as a decreasing function of the travel time plus the level of congestion at the preventive health-care facility, modelled as an $\mathbf{M} / \mathbf{M} / 1$ queue. We investigate models with both coverage and participation objectives in this paper. Verter and Lapierre (2002) introduce a binary integer programming model to find the optimal locations of preventive health-care facilities, which maximizes participation. Their model assumes that each population centre is assigned to the nearest facility and there is a minimum workload requirement for open facilities. Our model considers the effect of these constraints as well.

It is clear that different stakeholders involved in healthcare facility location problems may wish to optimize different objectives. For instance, patients would like to minimize their travel times while local governments would like to allocate as many people as possible to a health centre, or minimize the costs associated with establishing the facilities. In most of the health-care facility location applications, ensuring efficiency and equity are conflicting objectives since the number of facilities to be located is bounded due to budget and capacity constraints. A solution that compromises these conflicting objectives may help a central decision maker in developing effective and sustainable public health-care systems. In this context, Bruni et al (2006) propose a binary integer programming model to locate transplant centres by using an objective function incorporating both total travel distance and maximum waiting list size, which is the equity criterion forced to decrease by the model. Furthermore, Gu et al (2010) analyse a bi-objective model with the objectives of maximizing coverage and maximizing participation. They consider the problem of locating preventive health-care centres by proposing a new accessibility measure incorporating travel distances, catchment areas, and participation. Farinha et al (2008) discuss design of health facility networks with the objectives of quality and efficiency. Smith and Harper (2010) develop a bi-criteria model considering equity/efficiency for location of community clinics. For a detailed review of multi-objective facility location modes, the interested readers are referred to Current et al (1990).

The traditional facility location literature is limited in models that consider the assignment of resources, or the allocation of capacities to facilities. Ruth (1981) formulates a mixed integer programming model to determine optimal allocation of hospital beds in a given hospital network, with the option of extending the network. Güneş and Yaman (2010) develop a model to optimize re-allocation of beds between existing facilities. A main contribution of our model is that the optimal distribution of the family physicians is considered in addition to the optimal locations of the FHCs. Since each physician has a limited capacity of patients, this implies that the capacity of each facility is also a decision variable. This also allows us to consider the physician interests in the solutions.

\section{Problem description}

Our model takes the perspective of the central planner, while addressing the patient and physician preferences with different modelling choices and performance measures. This section discusses how our model addresses these different stakeholders' preferences.

\subsection{The central planner's objective}

The central planner for our problem is the local government, searching for a good solution to the planning problem. The main concern for the central planner is to maximize access of the population to primary health care, which is the objective function for our model. In this paper we focus on geographical barriers of access to health-care facilities. There are other barriers such as financial and cultural barriers, which are out of the scope of our discussion.

In this paper we use two measures for access as the objective function. First, the classical coverage, that is, the percentage of population assigned to a facility within a maximum travel distance, is maximized. Second, an alternative measure for access is used, which is a decreasing function of distance to facility. Classical coverage objectives are indifferent between any two points within the covered region, and therefore, do not address the distance concern. We define a participation function that decays with distance (Shuman et al, 1973; Zhang et al, 2009) to measure patient's access to primary care. Such an access function can be considered as a proxy for the usage of health-care facilities, as it is well-known that the probability of a person visiting a facility decreases with distance (Andersen and Newman, 1973; Zhang et al, 2009).

\subsection{Physician preferences}

According to studies done among primary care physicians, having a sufficient income and manageable workload are the most important factors that improve physician satisfaction, along with the ability to provide a high quality service, working in a collegial environment and obtaining 
professional support (Buchbinder et al, 2001; Lepnurm et al, 2007). Moreover, equity between colleagues is found to be positively correlated with satisfaction (Lepnurm et al, 2007). Accordingly, we identify the following as the criteria of concern for physicians: physician workload, physician income, professional support from colleagues, and equity among physicians in workload and income. The model addresses these factors using the following mechanisms:

- Income and workload: When a capitation-based contract is used, physician income is based on the panel size. With fee for service contracts, panel size indirectly determines income, since the demand is an increasing function of the panel size. Therefore, the income and workload-related concerns can be addressed with panel size constraints in general. A lower bound on panel size guarantees a minimum income level for physicians, and an upper bound restricts their workload.

- Equity: Equity concern is addressed via two mechanisms: First, the population assigned to an FHC is allocated equally between the physicians working at that health centre. Second, we use "the standard deviation of patient panel sizes' as a proxy for equitable distribution of income, and let the panel size per physician vary in a small range in order to control this value. This can be ensured by setting the lower and upper bounds for panel size per physician accordingly.

- Professional support and a collegial environment: This criterion is difficult to model, since inter-personal relationships is a key determinant of collegial environment. However, existence of group practice provides more opportunity to obtain professional support. Therefore, this criterion can be addressed by enforcing a lowerbound on the number of doctors working in the FHC.

In summary, our model addresses the physician concerns by: (1) explicitly modelling physician assignments to facilities, (2) imposing lower and upper bounds on the number of patients assigned to a physician, (3) imposing lower bounds on the number of physicians assigned to a facility. Table 1 provides a list of the critical factors that affect physician satisfaction and our operationalization of those factors.

\subsection{Patient preferences}

We focus on two main concerns that are important for patients: Access-related concerns such as travel distance and practice size, and equity-related concerns such as maximum travel distance.

- Distance to facilities: Previous research has shown that patients' utilization of health-care facilities decreases with distance and time (Andersen and Newman, 1973; Zhang et al, 2009). Therefore, the most important
Table 1 Proposed model parameters for factors that affect physician satisfaction

\begin{tabular}{ll}
\hline Factor & Parameter \\
\hline Income & Lower bound on panel size \\
Professional support from & FHC size (number of doctors \\
colleagues & per FHC) \\
Workload & Upper bound on panel size \\
$\begin{array}{l}\text { Equity of income distribution } \\
\text { among colleagues }\end{array}$ & $\begin{array}{l}\text { Range of panel sizes } \\
\text { (upperbound-lowerbound) }\end{array}$ \\
\hline
\end{tabular}

concern for patients is the proximity of the facility, which is also a common measure of access ${ }^{1}$. We use the average travel distance as a performance measure to represent patients' preferences. Moreover, decreasing the maximum allowable distance $\left(t_{\max }\right)$ is used as a mechanism in favour of patients.

- Practice size: It has been shown that patients prefer a smaller panel size at the general practice they attend (Baker and Streatfield, 1995). This would improve satisfaction of patients with accessibility and availability of medical care. This can be ensured by appropriate bounds on panel size in the model.

- Nearest assignment: Patients typically prefer the nearest facility to obtain service, and if there is an open facility that is within a shorter distance than the facility to which they are assigned to, they may prefer to patronize it. Therefore, a plan addressing patient preferences need to allocate them to the nearest open facility. This is satisfied by adding constraints to ensure allocating patients to the nearest open facility. This would weaken any incentives of patients to change their assigned health centres, and thus the solution would more likely be accepted by the patients.

- Equity: Equity is considered as an important concept and has been one of the major objectives of national health systems. Patients prefer that they do not travel longer distances than their neighbours. Therefore, patients at the same population centre should be assigned to facilities within the same distance. To maintain fair access between patients, we do not let partial assignment of a population centre, that is, all patients in a population centre should be assigned to the same facility. This ensures that all patients in a population zone travel the same distance to seek health service. The model uses single assignment constraints to ensure this. Moreover, we use the maximum travel distance, which is commonly used in the literature as a measure of equity of the solution.

\footnotetext{
${ }^{1}$ A more general approach could define access in terms of a preference function, which incorporates factors such as attractiveness of the location, or availability of public transportation, in addition to proximity.
} 
Table 2 Proposed model parameters for factors that affect patient satisfaction

\begin{tabular}{ll}
\hline Factor & Parameter \\
\hline Access & $\begin{array}{l}\text { Upper bound on distance to facilities } \\
\text { Upper bound on panel size } \\
\text { Nearest assignment constraint }\end{array}$ \\
Equity & $\begin{array}{l}\text { Single assignment constraint } \\
\text { Maximum travel distance performance }\end{array}$ \\
\hline
\end{tabular}

Table 2 provides a list of the critical factors that affect patient satisfaction and our operationalization of those factors.

\section{Model formulation}

Let $N$ be the set of population zones and $M$ be the set of possible locations for FHCs. For population zone $i \in N$, let $d_{i}$ be the population of zone $i$ and let $t_{i j}$ be the distance from zone $i$ to centre at location $j \in M$. We define $M_{i} \subseteq M$ to be the set of locations for FHCs that are within an acceptable distance or travel time $t_{\max }$ from population zone $i \in N$, that is, $M_{i}=\left\{j \in M: t_{i j} \leqslant t_{\max }\right\}$ and say that a person living in zone $i$ is covered if he/she is assigned to an FHC in set $M_{i}$.

We locate $p$ FHCs and allocate $q$ physicians. If an FHC is located, then at least $k$ physicians should be allocated to this facility. Due to the physical capacities, an FHC can host at most $\kappa$ physicians. The panel size for each physician should be not less than $l$ and not more than $u$.

We also let $\sigma_{i j}$ be the probability that a person from zone $i$ seeks services of his/her family physician if he/she is assigned to the FHC at location $j \in M$ and set $\sigma_{i j}=1-t_{i j} / t_{\max }$ if $t_{i j}$ is less than or equal to $t_{\max }$ and $\sigma_{i j}=0$ otherwise.

We define the following decision variables. For $j \in M, y_{j}$ is one if an FHC is located at location $j$ and zero otherwise and $z_{j}$ is the number of family physicians at this centre. The variable $x_{i j}$ takes value one if the population zone $i \in N$ is allocated to the FHC at location $j \in M$ and takes value zero otherwise.

Any vector satisfying the constraints below is a possible solution for our planning problem:

$$
\begin{gathered}
\sum_{j \in M} x_{i j}=1 \quad \forall i \in N \\
\sum_{j \in M} y_{j}=p \\
\sum_{j \in M} z_{j}=q
\end{gathered}
$$

$$
\begin{gathered}
l z_{j} \leqslant \sum_{i \in N} d_{i} x_{i j} \leqslant u z_{j} \quad \forall j \in M \\
k y_{j} \leqslant z_{j} \leqslant \kappa y_{j} \quad \forall j \in M \\
x_{i j} \in\{0,1\} \quad \forall i \in N, j \in M \\
z_{j} \in \mathbb{Z}_{+} \quad \forall j \in M \\
y_{j} \in\{0,1\} \quad \forall j \in M .
\end{gathered}
$$

Constraints (1) ensure that each population zone is served by a single FHC. The number of FHCs is $p$ and the number of family physicians is $q$ due to constraints (2) and (3). Constraints (4) impose lower and upper bounds on the number of people assigned to each family physician. Constraints (5) state that the number of family physicians at a centre should be at least $k$ and at most $\kappa$ if a centre is located here and no physician works here if a centre is not located. Finally, constraints (6)-(8) are variable restrictions.

For a given solution, the total coverage, that is, the number of people covered, is

$$
\sum_{i \in N} \sum_{j \in M_{i}} d_{i} x_{i j}
$$

the expected participation is

$$
\sum_{i \in N} \sum_{j \in M} d_{i} \sigma_{i j} x_{i j}
$$

and the total distance to be traveled by patients is

$$
\sum_{i \in N} \sum_{j \in M} d_{i} t_{i j} x_{i j}
$$

We obtain the model MC by maximizing coverage (9) and the model MP by maximizing participation (10) over the constraints (1)-(8). As MC typically has alternate optima, we solve the model MC-D to find a solution with minimum total distance among the solutions with maximum coverage. In this model, we minimize the function (11) subject to constraints (1)-(8) and the constraint $\sum_{i \in N} \sum_{j \in M_{i}} d_{i} x_{i j} \geqslant \beta$ where $\beta$ is the optimal value of the model MC.

If allocation to the nearest open facility is desired, then the following set of constraints can be added to the model (see, eg, Cánovas et al, 2007):

$$
\sum_{j \in M: t_{i j}>t_{i k}} x_{i j}+y_{k} \leqslant 1 \quad \forall i \in N, k \in M .
$$

These constraints ensure that for a given zone $i \in N$, if a facility at location $k \in M$ is open, then $i$ is not assigned to any facility whose distance to $i$ is more than the distance between $i$ and $k$. 


\section{An illustrative case study}

In this section, the proposed model is implemented using data from Sakarya, a small province in the north-west region of Turkey. Data used for the application of the model consist of 27 candidate locations for FHCs and 989 population centres, which are on the most crowded roads in the centre of the city. Figure 1 shows the road segments and candidate locations used in our analysis on the map of Sakarya. We model the population centres as the centre of the road segments, and the population size is the total size of the population living around the road segments to implement our model.

Our aim in this section is to demonstrate the use of our model with varying parameter values and obtain insights on the interactions of the objectives of the two stakeholders described in Section 1, as well as exploring the effect of increasing the number of facilities and physicians on different objectives. In addition to the base case, which represents the current solution approach in Sakarya, we analyse different scenarios that aim to address patient and physician preferences. The scenarios are labelled based on the stakeholder focus and the objective function used. For example, 'Patient-C' refers to a scenario addressing patient preferences and maximizes coverage objective, while 'Physician-P' is a scenario addressing physician preferences and maximizes participation. In addition, scenario 'Base-C' represents the current solution approach in Sakarya, and

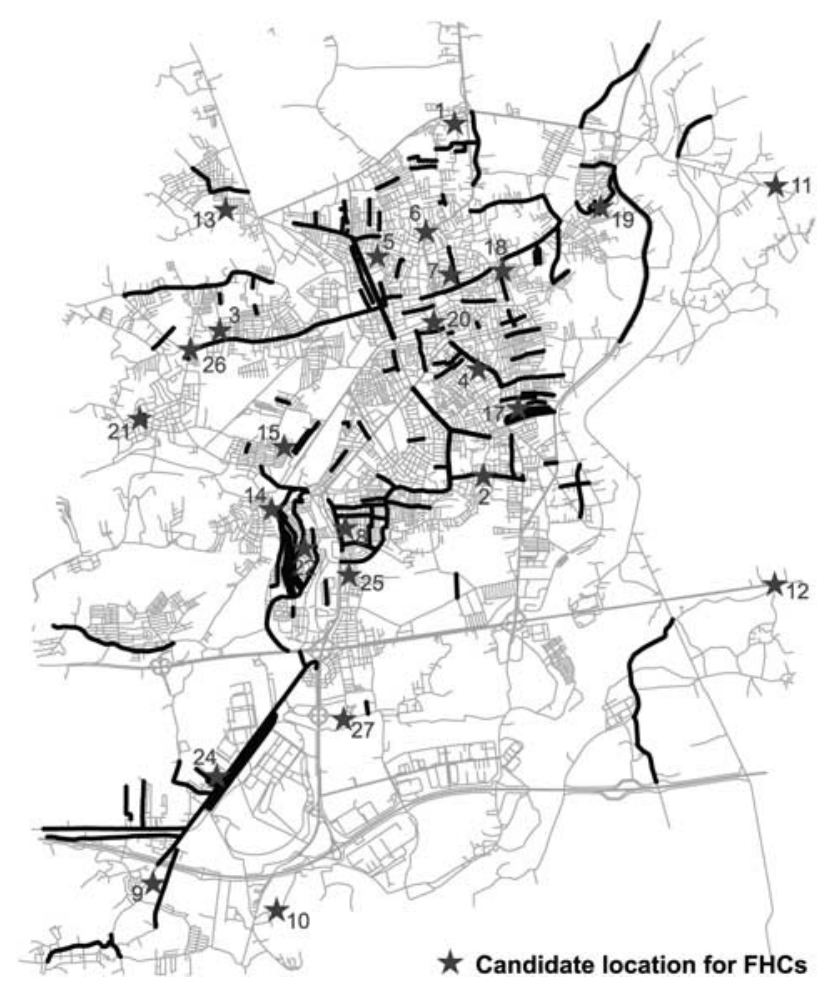

Figure 1 Road segments and candidate locations used in the analysis are shown on the map of Sakarya.
Table 3 Notation and parameter values used in numerical experiments

\begin{tabular}{lll}
\hline Parameter & Description & Values \\
\hline$t_{\max }$ & $\begin{array}{l}\text { Maximum distance for } \\
\text { coverage }\end{array}$ & $2,3 \mathrm{~km}$ \\
$u$ & $\begin{array}{l}\text { Upper bound on panel size } \\
l\end{array}$ & 4000 \\
$p$ & $\begin{array}{l}\text { Lower bound on panel size } \\
\text { Number of FHCs to be } \\
\text { opened }\end{array}$ & $2000,2500,3000$ \\
$q$ & $\begin{array}{l}\text { Number of physicians to } \\
\text { be hired }\end{array}$ & $15,17,19$ \\
$k$ & $\begin{array}{l}\text { Minimum number of } \\
\text { physicians in a FHC } \\
\text { Maximum number of } \\
\text { physicians }\end{array}$ & 1,2 \\
in a FHC & 5 \\
Allocation & Allocation constraints & free, nearest \\
\hline
\end{tabular}

Table 4 Definition of scenarios with the parameter values fixed for each scenario

\begin{tabular}{lcrrll}
\hline Scenario & $t_{\max }$ & \multicolumn{1}{c}{$l$} & $\kappa$ & Allocation & Objective \\
\hline Base-C & 3 & 2000 & 1 & Free & Coverage \\
Base-P & 3 & 2000 & 1 & Free & Participation \\
Physician-C & 3 & $2500-3000$ & 2 & Free & Coverage \\
Physician-P & 3 & $2500-3000$ & 2 & Free & Participation \\
Patient-C & 2 & 2000 & 1 & Nearest & Coverage \\
Patient-P & 2 & 2000 & 1 & Nearest & Participation \\
All-C & 2 & $2500-3000$ & 2 & Nearest & Coverage \\
All-P & 2 & $2500-3000$ & 2 & Nearest & Participation \\
\hline
\end{tabular}

scenario 'All' represents a solution aiming to address all concerns. Table 3 shows the notation and the range of parameter values used. Table 4 shows the parameter values that are fixed for each scenario. Note that the nearest assignment constraints defined by Constraints (12) are added only when the assignment is 'nearest'. On the other hand, Constraints (4) and (5) are included for all scenarios with different parameters, based on the dominant stakeholder preferences.

The possible ranges for model parameters were set in discussion with the city health authorities, who were in charge of planning the FHCs in preparation for the implementation of family practice in the city in $2010^{2}$.

If the objective for a particular scenario is to maximize coverage, we first solve model MC. We then solve MC-D to find a solution with minimum total distance among the solutions with maximum coverage. In the sequel, we report the results for these minimum distance solutions (found by solving MC-D) for the coverage objective. If the objective is to maximize participation, then we solve the model MP.

\footnotetext{
${ }^{2}$ This initiative has been one part of a comprehensive health reform in Turkey.
} 
Table 5 Results for the scenario base-C: $l=2000, k=1$ and $t_{\max }=3$

\begin{tabular}{|c|c|c|c|c|c|c|c|c|}
\hline$p$ & $q$ & $\% \operatorname{cov}$ & $\%$ part & Ave dist & Max dist & Locations of health centres & Max-min load & Std dev load \\
\hline \multirow[t]{3}{*}{4} & 15 & 89.00 & 37.37 & 2.07 & 8.92 & $3,8,9,18$ & 1816.09 & 644.48 \\
\hline & 17 & 89.00 & 39.96 & 2.09 & 9.05 & $3,8,9,18$ & 1999.16 & 811.28 \\
\hline & 19 & 89.00 & 36.98 & 2.29 & 12.20 & $3,8,9,18$ & 1868.67 & 768.61 \\
\hline \multirow[t]{3}{*}{6} & 15 & 95.26 & 53.10 & 1.51 & 7.80 & $3,5,8,9,17,19$ & 864.39 & 305.61 \\
\hline & 17 & 95.26 & 53.10 & 1.51 & 7.80 & $3,5,8,9,17,19$ & 1563.88 & 596.31 \\
\hline & 19 & 95.26 & 53.10 & 1.51 & 7.80 & $3,5,8,9,17,19$ & 1563.88 & 531.53 \\
\hline \multirow[t]{3}{*}{8} & 15 & 96.41 & 53.87 & 1.61 & 14.74 & $3,5,9,12,14,17,19,25$ & 1999.17 & 576.00 \\
\hline & 17 & 96.41 & 54.13 & 1.60 & 14.74 & $3,5,9,12,14,17,19,25$ & 1942.46 & 688.00 \\
\hline & 19 & 96.41 & 54.13 & 1.60 & 14.74 & $3,5,9,12,14,17,19,25$ & 1620.82 & 522.90 \\
\hline \multirow[t]{3}{*}{10} & 15 & 96.65 & 57.80 & 1.49 & 14.74 & $3,5,8,9,12,13,14,17,19,24$ & 1999.96 & 672.84 \\
\hline & 17 & 96.65 & 58.34 & 1.47 & 14.74 & $3,5,8,9,12,13,14,17,19,24$ & 1530.83 & 501.84 \\
\hline & 19 & 96.65 & 58.34 & 1.47 & 14.74 & $3,5,8,9,12,13,14,17,19,24$ & 1407.64 & 478.21 \\
\hline \multirow[t]{3}{*}{12} & 15 & 96.65 & 62.71 & $1.34^{\mathrm{a}}$ & 14.74 & $1,3,5,8,9,12,13,17,18,19,22,24$ & 1999.94 & 681.99 \\
\hline & 17 & 96.65 & 62.97 & 1.33 & 14.74 & $1,3,5,8,9,12,13,17,18,19,22,24$ & 1999.94 & 554.79 \\
\hline & 19 & 96.65 & 62.99 & 1.33 & 14.74 & $1,3,5,8,9,12,13,17,18,19,22,24$ & 1407.64 & 404.06 \\
\hline
\end{tabular}

${ }^{\mathrm{a}}$ Best solution, final gap in terms of total distance less than $0.002 \%$.

For each scenario, we report the following performance measures: the percentage coverage (cov), the percentage participation (part), average distance per patient (ave dist), the maximum distance ( $\max$ dist), the locations of the FHCs, the difference between the maximum and the minimum panel size (max-min), and the standard deviation of the panel sizes (std dev).

In the following, we first discuss the solution performance of each scenario for varying number of facilities and number of physicians ( $p$ and $q$ ), in Sections 5.1-5.4. Finally in Section 5.5, a comparison and discussion of scenarios are presented. The aim of the discussion here is to provide insights on the effects of resource investments and on choosing a good set of problem parameters for this primary health-care planning problem with multiple stakeholders.

\subsection{Base case with coverage objective}

The base case scenario represents the situation in Sakarya, where the parameters are set as chosen by city health officers. Please refer to Table 4 for the complete definition of the base case scenarios. Here, the scenario Base-C represents the situation in Sakarya. Note that the constraints on number of patients per physician and number of physicians per FHC limit the feasible values for the number of facilities $p$ and the number of doctors $q$. For example if $p=4$, the only feasible values of $q$ are 15, 17, and 19, since we can allocate at most five physicians to an FHC. Table 5 presents all performance measures for the optimal solutions for the base case. Here we summarize our observations from
Table 5, focusing on the effect of $p$ and $q$ on relevant performance measures.

For the Base-C scenario, the maximum coverage value is $96.65 \%$, which can be achieved with 10 FHCs and 15 physicians. As expected, the main determinant of coverage is the number of facilities. However, marginal benefit of adding more facilities is decreasing: increasing $p$ from 4 to 6 increases coverage by $7 \%$, while the maximum coverage values for $p=10$ and $p=12$ are equal.

Opening more facilities improves coverage; however, that may come at the expense of increasing the maximum distance travelled by patients. For one case, when $p$ is increased from 6 to 8 , average distance travelled also increases.

An interesting observation is the negative effect of increasing $q$, the number of physicians, on participation and travel distance measures. That is, the performance deteriorates by adding resources. When there are few facilities, adding physicians increases the average travel distance and decreases participation. This counter-intuitive effect is a result of the lower bound on the number of patients allocated to a physician (2000 for this case) and the upper bound on the number of physicians allocated to each FHC (five for this case). When a physician is added, it may become necessary to assign some patients to a farther FHC so as to satisfy the lower bound for that physician. This is an example of how considering physician preferences would change the optimal solutions.

The standard deviation of panel sizes, a measure of equity between physicians, is affected by both $p$ and $q$. For few facilities, increasing the number of physicians may increase standard deviation (see for example $p=4, q=15$ 
versus $q=17$ ). This is mainly because of the upper bounds on the number of patients allocated to each physician. For small values of $p$ and $q$, the panel sizes are quite close to the upper bounds, which results in low standard deviation. However, when $p$ is larger, standard deviation of panel sizes decreases with more physicians. In this case with more physicians, the lower bound constraint on the panel sizes becomes more and more tight, which, again, results in lower standard deviations. To summarize, it becomes easier to maintain equity between physicians in terms of the panel sizes with many facilities and many physicians or with few facilities and few physicians.

Finally, in the optimal solutions the same locations appear most of the time. This suggests that in practice, opening few facilities and adding new ones as necessary could be a plausible approach, which would not result in a very poor solution.

\subsection{Base case with participation objective}

In this scenario, we solve the problem with Base-C case parameters, using the expected participation objective instead of the total coverage objective. The performance of the optimal solutions for this case can be seen in Table 6 .

As expected, Base-P scenario has lower total coverage and larger expected participation than the Base-C case for all $p, q$ pairs. An interesting observation is that while the total coverage decreases by only $3.26 \%$, the expected participation increases by $9.36 \%$ on the average. This is mainly due to the fact that the expected participation is always less than or equal to the total coverage for every feasible solution.

Furthermore, the average travel distance is always lower in the Base-P scenario than in the Base-C case, with a $14.74 \%$ average decrease. This is mainly because of the travel time component in the expected participation formulation (10). It should be also noted that Base-P provides the largest expected participation and the lowest average travel distance values for $p, q$ pairs that are feasible for all scenarios analysed in this paper.
These results suggest that significantly better solutions in terms of expected participation and average travel distance can be obtained by maximizing the expected participation instead of total coverage.

\subsection{Addressing physician preferences}

Our next scenario reflects the concerns related with physician preferences: To avoid assigning a single physician to an FHC (so that physicians have professional support from colleagues), we set the minimum number of physicians to be assigned to a facility, $k=2$. We also increase the lower bound on the panel size per physician, $l$ to 2500 and 3000 , in order to improve equity between physicians and ensure higher wages per physician. The remaining parameters are varied. Since the total population size is 50489 , only $q=15$ is feasible with a lower bound of 3000 patients per physician. That restriction also restricts the number of facilities, $p$, to 4 and 6 only, as at least two physicians should be assigned to each FHC. Similarly, the feasible set of $p$ and $q$ values for $l=2500$ are found as $p \in\{4,6,8\}$ and $q \in\{15,17,19\}$ due to lower bound on the number of physicians per facility, $k=2$.

The performance of optimal solutions for this scenario can be seen in Tables 7 and 8 , for the coverage and participation objectives as access measure, respectively. Maximum coverage in this case is $96.18 \%$ of the population, slightly lower than the Base-C case. This can be achieved with $p=8$ facilities and $q=19$ physicians. In this case, a facility (number 12), which is distant to the centre, is not opened. This is because only one physician would be required at that centre due to the size of the population that would be assigned to that facility. As a consequence, some individuals cannot be covered within the maximum travel distance of $3 \mathrm{~km}$, and the total coverage is slightly lower than the Base-C case. Similar to the Base-C case, we observe that increasing the number of physicians, $q$, may decrease coverage.

This scenario aims at improving physician satisfaction by increasing average income per physician and by

Table 6 Results for scenario base-P: $l=2000, k=1$ and $t_{\max }=3$

\begin{tabular}{|c|c|c|c|c|c|c|c|c|}
\hline$p$ & $q$ & $\% \operatorname{cov}$ & $\%$ part & Ave dist & Max dist & Locations of health centres & Max-min load & Std dev load \\
\hline \multirow[t]{3}{*}{4} & 15 & 85.37 & 46.64 & 1.80 & 7.80 & $5,8,17,24$ & 245.95 & 109.96 \\
\hline & 17 & 85.37 & 46.64 & 1.80 & 7.80 & $5,8,17,24$ & 974.98 & 434.04 \\
\hline & 19 & 85.37 & 46.64 & 1.82 & 10.44 & $5,8,17,24$ & 1496.05 & 539.92 \\
\hline \multirow[t]{3}{*}{6} & 15 & 90.81 & 54.33 & 1.50 & 7.80 & $3,5,8,17,18,24$ & 915.18 & 338.76 \\
\hline & 17 & 90.81 & 54.33 & 1.49 & 7.80 & $3,5,8,17,18,24$ & 1133.50 & 385.88 \\
\hline & 19 & 90.81 & 54.33 & 1.49 & 7.80 & $3,5,8,17,18,24$ & 943.32 & 381.68 \\
\hline \multirow[t]{3}{*}{8} & 15 & 92.38 & 59.61 & 1.28 & 7.80 & $1,3,5,8,17,18,22,24$ & 1017.22 & 342.07 \\
\hline & 17 & 92.38 & 59.61 & 1.28 & 7.80 & $1,3,5,8,17,18,22,24$ & 1828.51 & 629.79 \\
\hline & 19 & 92.38 & 59.61 & 1.28 & 7.80 & $1,3,5,8,17,18,22,24$ & 1515.29 & 491.95 \\
\hline
\end{tabular}


Table 7 Results for scenario physicians-C: $l=2500$ and $3000, k=2$ and $t_{\max }=3$

\begin{tabular}{lccccccrrr}
\hline$l$ & $p$ & $q$ & $\%$ cov & $\%$ part & Ave dist & Max dist & Locations of health centres & Max-min load & Std dev load \\
\hline 2500 & 4 & 15 & 89.00 & 37.24 & 2.07 & 9.05 & $3,8,9,18$ & 1499.56 \\
2500 & 4 & 17 & 89.00 & 36.98 & 2.25 & 11.61 & $3,8,9,18$ & 1370.35 \\
2500 & 4 & 19 & 88.46 & 38.76 & 2.31 & 13.35 & $9,15,17,18$ & 596.86 \\
2500 & 6 & 15 & 95.26 & 51.73 & 1.55 & 7.8 & $3,5,8,9,17,19$ & 1496.52 \\
2500 & 6 & 17 & 95.26 & 51.73 & 1.55 & 7.8 & $3,5,8,9,17,19$ & 880.86 \\
2500 & 6 & 19 & 95.26 & 51.66 & 1.55 & 7.8 & $3,5,8,9,17,19$ & 775.65 \\
2500 & 8 & 17 & 96.18 & 47.03 & 1.66 & 8.04 & $1,3,9,13,14,17,19,25$ & 1499.9 & 262.73 \\
2500 & 9 & 19 & 96.18 & 47.2 & 1.65 & 7.8 & $1,3,9,13,14,17,19,25$ & 551.8 \\
3000 & 4 & 15 & 89.00 & 36.96 & 2.13 & 9.05 & $3,8,9,18$ & 99.77 \\
3000 & 6 & 15 & 95.26 & 50.47 & 1.59 & 7.80 & $3,5,8,9,17,19$ & 99.07 \\
\hline
\end{tabular}

Table 8 Results for scenario physicians-P: $l=2500$ and $3000, k=2$ and $t_{\max }=3$

\begin{tabular}{|c|c|c|c|c|c|c|c|c|c|}
\hline$l$ & $p$ & $q$ & $\% \operatorname{cov}$ & $\%$ part & Ave dist & Max dist & Locations of health centres & Max-min load & Std dev load \\
\hline 2500 & 4 & 15 & 46.64 & 85.37 & 1.8 & 1.8 & $5,8,17,24$ & 337.14 & 135.07 \\
\hline 2500 & 4 & 17 & 46.64 & 85.37 & 1.8 & 7.8 & $5,8,17,24$ & 967.47 & 430.02 \\
\hline 2500 & 4 & 19 & 46.64 & 85.37 & 2.06 & 11.53 & $5,8,17,24$ & 574.86 & 249.69 \\
\hline 2500 & 6 & 15 & 54.33 & 90.74 & 1.61 & 9.05 & $3,5,8,17,18,24$ & 1496.68 & 507.26 \\
\hline 2500 & 6 & 17 & 54.33 & 90.74 & 1.6 & 9.05 & $3,5,8,17,18,24$ & 819.39 & 213.3 \\
\hline 2500 & 6 & 19 & 54.33 & 90.74 & 1.63 & 9.05 & $3,5,8,17,18,24$ & 528.39 & 199.09 \\
\hline 2500 & 8 & 17 & 59.57 & 91.45 & 1.56 & 12.44 & $1,3,5,8,17,18,22,24$ & 1185.89 & 432.96 \\
\hline 2500 & 8 & 19 & 59.52 & 91.45 & 1.56 & 12.44 & $1,3,5,8,17,18,22,24$ & 1185.89 & 505.15 \\
\hline 3000 & 4 & 15 & 46.64 & 85.37 & 1.8 & 7.8 & $5,8,17,24$ & 245.94 & 109.96 \\
\hline 3000 & 6 & 15 & 54.33 & 90.74 & 1.68 & 9.05 & $3,5,8,17,18,24$ & 943.95 & 342.89 \\
\hline
\end{tabular}

decreasing the standard deviation of the panel sizes. Our results suggest that standard deviation decreased for some cases; however, the reverse is also possible (eg, for $p=6, g=5$, surprisingly, the Base-C case has lower standard deviation). One explanation for this result is the fact that the minimum number of physicians per facility is increased to two (to ensure professional support for physicians). This restriction could be the reason for the increased standard deviation per physician. This result suggests that there is a trade-off between these two preferences of the physicians. Ensuring equity and professional support at the same time may not be possible. Another important observation is that although both Physician-P and Physician-C scenarios have lower standard deviations compared to the Base- $\mathrm{C}$ case, Physician-P scenario achieves more improvement. Thus, it can be concluded that the expected participation objective is a better access measure than the total coverage to satisfy the physicians' preferences.

Our results also illustrate the trade-offs between the preferences of the physicians and the central decision maker, and between the preferences of the physicians and the patients. Note that both Physician-C and Physician-P scenarios have lower total coverage values for all $p$ and $q$ values compared to the Base- $\mathrm{C}$ case, and Physician-C scenario always provides lower average travel distances than the Base-C case.
Modifying the model to make the solution more desirable for the physicians may hurt accessibility objective, by restricting the number of facilities that can be opened. However, our results show that the decrease in accessibility is not substantial. Even though at most eight facilities can be established and 19 doctors can be allocated to the located facilities, the maximum achievable total coverage decreases only by $0.4 \%$ (from $96.65 \%$ to $96.18 \%$ ) in Physician-C scenario. Moreover, the expected participation increases (for example, see the case $p=4$ and $q=19$ ).

\subsection{Addressing patient preferences}

Patients' scenario emphasizes the travel distance concern of patients with a more ambitious coverage target, and also assigns patients to the nearest open FHC. Hence, we set the maximum travel distance $t_{\max }$ to $2 \mathrm{~km}$, and include the nearest allocation constraints (12) given in Section 3. All the other parameters are the same as the Base- $\mathrm{C}$ case. This scenario is solved for two objective functions, participation and coverage. Tables 9 and 10 show performance of optimal solutions measured as the maximum coverage, percentage of participation, the average distance, and maximum distance per patient for each $p, q$ pair.

First, we compare the Patient's scenario with the Base-C case based on the average and maximum travel distance 
Table 9 Results for scenario patients-P: $l=2000, k=1, t_{\max }=2$ and assignment to the nearest centre

\begin{tabular}{|c|c|c|c|c|c|c|c|c|}
\hline$p$ & $q$ & $\% \operatorname{cov}$ & $\%$ part & Ave dist & Max dist & Locations of health centres & Max-min load & Std dev load \\
\hline \multirow[t]{3}{*}{4} & 15 & 67.70 & 31.32 & 1.80 & 7.80 & $5,8,17,24$ & 337.14 & 135.07 \\
\hline & 17 & 67.70 & 31.32 & 1.80 & 7.80 & $5,8,17,24$ & 1191.68 & 472.35 \\
\hline & 19 & 62.76 & 29.74 & 2.19 & 8.53 & $5,7,8,17$ & 1915.09 & 803.72 \\
\hline \multirow[t]{3}{*}{6} & 15 & 77.46 & 39.57 & 1.50 & 7.80 & $5,8,17,18,22,24$ & 846.61 & 296.54 \\
\hline & 17 & 77.46 & 39.57 & 1.50 & 7.80 & $5,8,17,18,22,24$ & 1416.82 & 409.88 \\
\hline & 19 & 77.46 & 39.57 & 1.50 & 7.80 & $5,8,17,18,22,24$ & 1339.51 & 468.11 \\
\hline \multirow[t]{3}{*}{8} & 15 & 84.51 & 43.68 & 1.31 & 7.80 & $1,3,5,7,8,17,22,24$ & 866.81 & 278.85 \\
\hline & 17 & 82.54 & 45.14 & 1.32 & 7.23 & $2,3,5,8,17,18,22,24$ & 647.34 & 214.46 \\
\hline & 19 & 82.54 & 45.14 & 1.32 & 7.23 & $2,3,5,8,17,18,22,24$ & 909.67 & 322.51 \\
\hline \multirow[t]{3}{*}{10} & 15 & 86.97 & 46.69 & 1.21 & 7.80 & $3,4,5,8,9,17,18,19,22,24$ & 1253.54 & 494.45 \\
\hline & 17 & 87.27 & 49.05 & 1.15 & 7.23 & $1,2,3,5,8,17,18,19,22,24$ & 1249.22 & 387.49 \\
\hline & 19 & 86.83 & 49.46 & 1.18 & 7.23 & $1,2,3,5,8,15,17,18,22,24$ & 1814.88 & 540.00 \\
\hline \multirow[t]{3}{*}{12} & 15 & 88.59 & 48.72 & 1.17 & 7.80 & $3,4,5,7,8,9,14,17,18,19,22,24$ & 1692.37 & 535.18 \\
\hline & 17 & 91.17 & 51.44 & 1.08 & 7.23 & $1,2,3,5,8,9,14,17,18,19,22,24$ & 1484.91 & 477.67 \\
\hline & 19 & 88.98 & 52.68 & 1.08 & 7.23 & $1,2,3,5,8,15,17,18,19,20,22,24$ & 1097.63 & 366.94 \\
\hline
\end{tabular}

Table 10 Results for scenario patients-C: $l=2000, k=1, t_{\max }=2$ and assignment to the nearest centre

\begin{tabular}{|c|c|c|c|c|c|c|c|c|}
\hline$p$ & $q$ & $\% \operatorname{cov}$ & $\%$ part & ave dist & max dist & Locations of health centres & Max-min load & Std dev load \\
\hline \multirow[t]{3}{*}{4} & 15 & 68.69 & 27.24 & 1.86 & 7.80 & $6,8,9,17$ & 258.74 & 106.26 \\
\hline & 17 & 68.69 & 27.24 & 1.86 & 7.80 & $6,8,9,17$ & 928.54 & 398.63 \\
\hline & 19 & 65.64 & 26.44 & 2.24 & 8.56 & $2,5,14,18$ & 1009.89 & 472.99 \\
\hline \multirow[t]{3}{*}{6} & 15 & 81.37 & 34.90 & 1.53 & 7.80 & $5,8,9,14,17,19$ & 819.54 & 279.98 \\
\hline & 17 & 81.37 & 34.90 & 1.53 & 7.80 & $5,8,9,14,17,19$ & 1495.29 & 538.22 \\
\hline & 19 & 81.37 & 34.90 & 1.53 & 7.80 & $5,8,9,14,17,19$ & 1256.98 & 391.51 \\
\hline \multirow[t]{3}{*}{8} & 15 & 88.20 & 41.23 & 1.34 & 7.80 & $3,5,8,9,14,17,19,24$ & 1132.68 & 365.54 \\
\hline & 17 & 89.13 & 40.07 & 1.34 & 7.80 & $1,3,5,8,9,14,17,19$ & 1492.88 & 592.35 \\
\hline & 19 & 89.13 & 40.07 & 1.34 & 7.80 & $1,3,5,8,9,14,17,17,19$ & 1324.92 & 362.92 \\
\hline \multirow[t]{3}{*}{10} & 15 & 88.44 & 45.69 & 1.24 & 7.80 & $3,4,5,8,9,14,17,18,19,24$ & 1379.45 & 489.82 \\
\hline & 17 & 91.10 & 45.38 & 1.22 & 7.80 & $1,3,5,8,14,17,17,19,20,24$ & 1023.18 & 354.14 \\
\hline & 19 & 91.22 & 43.88 & 1.23 & 7.80 & $1,3,5,9,15,17,19,22,24,25$ & 1190.99 & 415.37 \\
\hline \multirow[t]{3}{*}{12} & 15 & 90.12 & 46.40 & 1.21 & 7.80 & $3,4,6,7,8,9,14,17,18,19,22,24$ & 1541.65 & 475.33 \\
\hline & 17 & 31.38 & 48.77 & 1.13 & 7.23 & $1,2,3,5,9,15,17,19,20,24,25$ & 1600.54 & 476.30 \\
\hline & 19 & 91.38 & 48.77 & 1.13 & 7.23 & $1,2,3,5,9,15,17,19,20,22,24,25$ & 1723.25 & 486.83 \\
\hline
\end{tabular}

measures. Note that the coverage criteria are different for these two scenarios ( $3 \mathrm{~km}$ versus $2 \mathrm{~km}$ ), therefore we cannot compare them on the basis of coverage or participation. However, travel distance, which is the most important criterion for patients, can be used as a basis for comparison of scenarios.

Patient- $\mathrm{C}$ scenario performs better than the Base-C case for $78 \%$ of all $p, q$ pairs in the average distance measure, and for $95 \%$ of all $p, q$ pairs in the maximum distance measure. The same observation holds with the Patient-P scenario for $95 \%$ and $100 \%$ of all instances, respectively.
Note that Patient-P scenario has always a lower average travel distance and a lower maximum distance than the Patient-C scenario. Moreover on the average, the expected participation is $12 \%$ higher in Patient-P scenario than in Patient-C scenario. This is achieved at the expense of only a $3.9 \%$ decrease in the total coverage. These observations clearly indicate that the expected participation is a better access measure to satisfy the patients' preferences.

Another interesting observation in favour of the expected participation objective concerns the standard 
deviation of the panel sizes. Patient-C scenario, with the coverage objective, achieves an average $27 \%$ decrease in standard deviation of panel sizes in $73 \%$ of all cases. On the other hand, the Patient-P scenario, using the participation objective, improves over the Base-C case in $82 \%$ of all cases, with an average improvement of $29 \%$.

Finally, the results show a similar pattern with changing values of $p$ and $q$. Increasing $q$ may have a detrimental effect on all performance measures considered, while increasing $p$ is always beneficial.

\subsection{Comparison of scenarios: exploring the trade-offs}

In this section the trade-offs between different objective values will be explored using the numerical results obtained in the previous sections. In addition, we combine the patient and physician preferences in a new scenario (Scenario All). Scenario All sets lower bound of panel size to 3000 , lower bound of physicians per facility to $2, t_{\max }$ to 2 , and incorporates the nearest allocation constraint. Thus, we provide a solution that addresses both the physician and patient preferences with scenario All.

Our objective is to provide insights on choosing a good set of problem parameters for this primary health-care delivery planning problem with multiple stakeholders and multiple criteria to be considered. We focus on two performance measures: patient satisfaction, measured by average travel distance, and physician satisfaction, measured by standard deviation of the panel sizes per physician.

In Figure 2, we plot performance of the scenarios on two dimensions: the average distance per patient on the vertical axis (representing the patient preferences) and the standard deviation of panel sizes on the horizontal axis

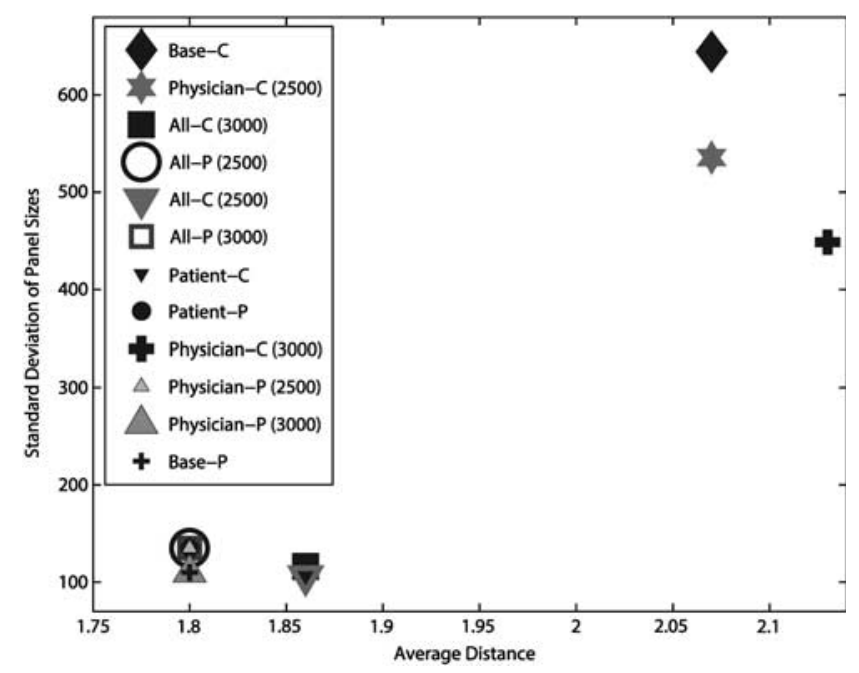

Figure 2 The performance of the scenarios in terms of average travel distance and standard deviation of panel sizes when $p=4$ and $q=15$. (representing the physician preferences). Note that the value of maximum coverage distance, $t_{\max }$, can be different for different scenarios, which makes the comparison of scenarios on participation or coverage measures an unfair one. Since the meaning of average travel distance is the same for all scenarios, a comparison based on a distance measure is meaningful.

Figure 2 shows that the Base-P, Physician-P, Patient-C, and All-C are on the efficient frontier and the Base-C case and Physicians-C Scenario are the worst performing scenarios for $p=4$ and $q=15$.

The efficient frontiers for all $p, q$ pairs are given in Table 11. If a scenario is not on the efficient frontier for a given $(p, q)$ pair, then the corresponding cell is left empty in Table 11 for that scenario. Otherwise, if a scenario is on the efficient frontier, then a number is shown, which indicates the position of the scenario on the efficient frontier. The scenarios on the efficient frontier are ordered in increasing average distance and decreasing standard deviation. For example, if number 1 is indicated for a particular scenario, this implies that this scenario is on the efficient frontier. Further, it has the minimum average distance and the maximum standard deviation among the scenarios on the efficient frontier. Finally, for a $(p, q)$ pair, the scenario with the maximum total coverage is indicated with an asterisk.

The average distance and standard deviation measures change as a function of the number of FHCs $(p)$, and the number of doctors $(q)$, as discussed in previous sections. When these resources are kept constant, one can decrease the standard deviation of panel sizes at a cost of increasing average distance (and vise versa), by choosing among the four scenarios, which are on the efficient frontier.

Note that the Base-C case is dominated by other scenarios for all $p, q$ pairs. In other words, we can always find a scenario that has less average travel distance and standard deviation of panel sizes. The fact that the Base-C scenario represents the real situation implemented in the province of Sakarya is an indication of the improvement potential by using our model. In addition, the scenarios with the largest total coverage on the efficient frontier have only $4.4 \%$ less total coverage than the Base-C case. This shows that a significantly better solution can be obtained without any increase in resource investments and any significant decline in the objective of the central decision maker.

Among the scenarios compared, Base-P, Physician-P, All-C, and All-P perform well in general and they are on the efficient frontier for almost all cases. The main reason for Base-P scenario to be on the efficient frontiers is that it generally produces very low average travel distances compared to the other scenarios. Similarly, All-C scenario appears on the efficient frontiers mostly due to lower standard deviations in this scenario. It is interesting that three out of these four scenarios have an expected 
Table 11 Efficient solutions for all $(p, q)$ pairs

\begin{tabular}{|c|c|c|c|c|c|c|c|c|}
\hline \multirow[t]{2}{*}{$p, q$ pairs } & \multicolumn{8}{|c|}{ Scenarios } \\
\hline & Base-C & Base-P & Patient-C & Patient-P & Physician-C & Physician- $P$ & All-C & All-P \\
\hline 4,15 & & $1^{*}$ & 2 & & & $1^{*}$ & 2 & \\
\hline 4,17 & & & 2 & & & $1^{*}$ & 2 & 1 \\
\hline 4,19 & & $1^{*}$ & & & & $2^{*}$ & 3 & 3 \\
\hline 6,15 & & & $2^{*}$ & 1 & & & $2 *, 3$ & 1 \\
\hline 6,17 & & 1 & & & $3^{*}$ & 4 & & 2 \\
\hline 6,19 & & 1 & & & $3 *$ & 5 & 4 & 2 \\
\hline 8,17 & & $1^{*}$ & & 2 & & & & \\
\hline
\end{tabular}

* The scenario with the maximum total coverage for each $(p, q)$ pair is indicated by an asterisk.

Note: Increasing numbers indicate increasing average distance.

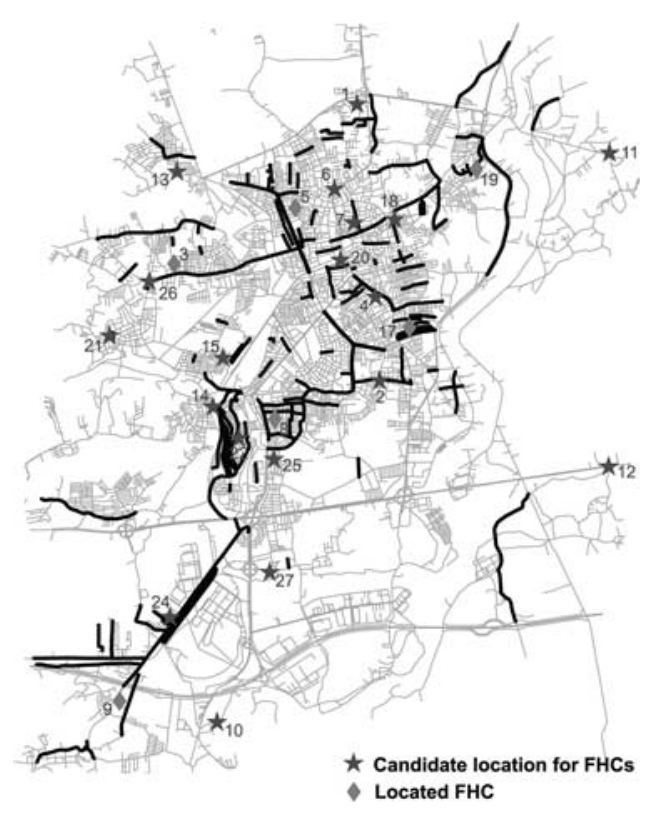

Figure 3 The optimal locations of the FHCs for the Base-C case and Physician-C scenarios when $p=6, q=15$.

participation objective and include the modifications to satisfy the physicians' preferences.

We present the optimal locations of FHC's in three scenarios to illustrate the differences in the solutions in Figures 3 and 4 . We observe that the optimal location for the Base-C case and Pysicians-C scenario, which are both dominated by other scenarios, are the same. The difference in the performance of these two scenarios is a result of the different allocation decisions. On the other hand, for Scenarios All-P, which incorporates all preferences and is on the efficient frontier, half of the locations are changed. Thus, the better performance is a result of both location and allocation decision in that case.

Our computational experiments with the Sakarya case demonstrate the multi-objective nature of the primary case network design problem. On the basis of our findings (summarized in Table 11), it is advisable for the planners to

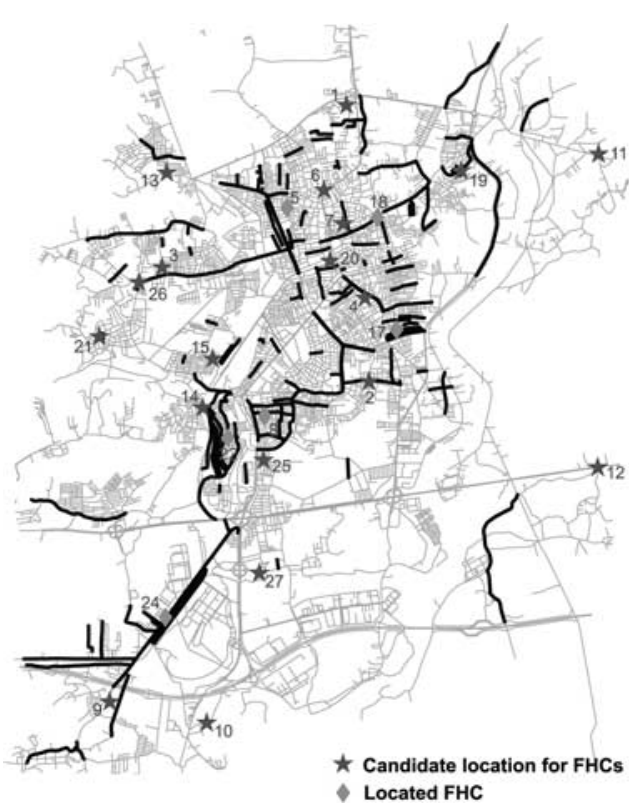

Figure 4 The optimal locations of the FHCs for the All-P scenario when $p=6, q=15$.

study the design problem under multiple scenarios so that all elements of the efficient frontier can be captured via the collection of the scenarios. For example, in the Sakarya case, solving the Base-P, Physician-P, All-P, and All-C scenarios seem to be sufficient to represent the efficient frontier of the possible configurations. Perhaps more importantly, the Base-C scenario-representing the current situation - is not a non-dominated solution.

\section{Summary and conclusions}

Health-care systems should be designed to best serve the needs of the population. If the physicians, the main agents of health-care delivery, are satisfied with the system, not only this important stakeholder of health systems would be satisfied but also the quality of medical care would 
improve, and the risk of lack of physicians would diminish. With this motivation, this paper presented a discrete location-allocation model for planning FHCs that aims to match physicians' and patients' preferences. The main contribution to the traditional facility location literature is the explicit modelling of allocation of physicians, and an analysis of models considering preferences of different stakeholders. This generated insights for future primary health-care facility modelling, by providing an understanding of the effect of problem parameters and constraints on different performance measures and the interactions between preferences of different stakeholders.

In summary, we make several interesting observations in this implementation of our model. First, the number of facilities that will be opened is the main determinant of access. Opening more facilities increases coverage of the population ${ }^{3}$. However, while improving coverage, the average distance a patient has to travel may increase as well. Second, a surprising observation is that increasing the number of doctors does not have a monotone effect on patient access. More doctors may make it difficult to allocate the minimum panel size for each doctor, hence it may decrease all access-related measures considered. Increasing the number of doctors in general improves equity among doctors, except for cases with very few facilities.

Furthermore, our results show that incorporating the nearest allocation constraints and using a participation objective as a measure of access improve performance in both patient and physician-related measures. We believe incorporating these characteristics would be beneficial in other health-care facility planning models as well.

Finally, for fixed amount of resource (ie, fixed number of physicians and facilities) there is a trade-off between patients' access-related measures and physician satisfaction measure of equity between physicians. Given relative importance of these two objectives, one could use the scenario that finds the right balance between them.

There are possible research directions that can build on this work. The approach in this paper can be extended by including the mobile service facilities or physicians, and incorporating the impact of distance to facilities on visit frequencies, which affect the congestion level in the healthcare centres. Empirical research can aim to elicit preferences of physicians and workers, which would lead to incorporating additional constraints or objective functions in the model. Finally it would be interesting to investigate robustness of our observations with different participation functions.

Acknowledgements - The fourth author was supported by a Discovery Grant from the Natural Sciences and Engineering Council (NSERC) of Canada and by the Global researcher Programme of the Scientific

\footnotetext{
${ }^{3}$ Note that there may be exceptional cases in which opening a facility may deteriorate coverage, depending on the location of candidate facilities. But this was not the case for our implementation.
}

and Technological Research Council of Turkey (TUBITAK). The authors are grateful to Burcin Bozkaya for providing the GIS-based population and road data for the Sakarya province. The authors also gratefully acknowledge Sakarya Provincial Health Department for the data and information provided.

\section{References}

Andersen R and Newman J (1973). Societal and individual determinants of medical care utilization in the United States. Milbank Memorial Fund Quarterly 51(1): 95-123.

Baker R and Streatfield J (1995). What type of general practice do patients prefer? Exploration of practice characteristics influencing patient satisfaction. British Journal of General Practice 45(401): 654-659.

Berman O and Krass D (2002). The generalized maximal covering location problem. Computers \& Operations Research 29(6): 563-581.

Bruni M, Conforti D, Sicilia N and Trotta S (2006). A new organ transplantation location-allocation policy: A case study of Italy. Health Care Management Science 9(2): 125-142.

Buchbinder S, Wilson M, Melick C and Powe N (2001). Primary care physician job satisfaction and turnover. American Journal of Managed Care 7(7): 701-716.

Cánovas L, García S, Labbé M and Marín A (2007). A strengthened formulation for the simple plant location problem with order. Operations Research Letters 35(2): 141-150.

Church R and ReVelle C (1974). The maximal covering location problem. Papers in Regional Science 32(1): 101-118.

Current J, Min H and Schilling D (1990). Multiobjective analysis of facility location decisions. European Journal of Operational Research 49(3): 295-307.

Daskin M and Dean L (2005). Location of health care facilities. In: Brandeau ML, Sainfort F and Pierskalla WP (eds). Operations Research and Health Care. International Series in Operations Research \& Management Science, Springer, Boston, USA, pp 43-76.

Farinha R, Duarte Oliveira M and de Sa A (2008). Networks of primary and secondary care services: How to organise services so as to promote efficiency and quality in access while reducing costs. Quality in Primary Care 16(4): 249-258.

Gladu F (2007). Perceived shortage of family doctors in Quebec: Can we do something about it? Canadian Family Physician 53(11): 1858.

Gu W, Wang X and McGregor S (2010). Optimization of preventive health care facility locations. International Journal of Health Geographies 9(1): Article 17.

Güneş E and Yaman H (2010). Health network mergers and hospital re-planning. Journal of the Operational Research Society 61(2): 275-283.

Jia H, Ordóñez F and Dessouky M (2007). A modeling framework for facility location of medical services for large-scale emergencies. IIE Transactions 39(1): 41-55.

Kindig D and Movassaghi H (1989). The adequacy of physician supply in small rural counties. Health Affairs 8(2): 63-76.

Lepnurm R, Dobson R, Backman A and Keegan D (2007). Factors associated with career satisfaction among general practitioners in Canada. Canadian Journal of Rural Medicine: The Official Journal of the Society of Rural Physicians of Canada 12(4): 217-230.

Macinko J, Starfield B and Shi L (2003). The contribution of primary care systems to health outcomes within organization for economic cooperation and development (OECD) countries, 1970-1998. Health Services Research 38(3): 831-865. 
MacKean P and Gutkin C (2003). Fewer medical students selecting family medicine. Can family practice survive? Canadian Family Physician 49(4): 408.

Marianov V and Taborga P (2001). Optimal location of public health centres which provide free and paid services. Journal of the Operational Research Society 52(4): 391-400.

Massachusetts Medical Society (2007). 2007 Massachusetts medical society physician workforce study, http://www.massmed .org/Content/NavigationMenu/NewsandPublications/Research ReportsStudies/PhysicianWorkforceStudy/workforce07.htm, accessed 28 June 2012.

O'Reilly K (2010). Health reform's next challenge: Who will care for the newly insured. American Medical News, 12 April.

Owen S and Daskin M (1998). Strategic facility location: A review. European Journal of Operational Research 111(3): 423-447.

Pong R and Pitblado J (2005). Geographic Distribution of Physicians in Canada: Beyond How Many and Where. Canadian Institute for Health Information: Ottawa, Canada.

Rahman S and Smith D (1999). Deployment of rural health facilities in a developing country. Journal of the Operational Research Society 50(9): 892-902.

Rais A and Viana A (2011). Operations research in healthcare: A survey. International Transactions in Operational Research 18(1): $1-31$.

Rourke J (2008). Increasing the number of rural physicians. Canadian Medical Association Journal 178(3): 322.
Ruth R (1981). A mixed integer programming model for regional planning of a hospital inpatient service. Management Science 27(5): 521-533.

Shuman L, Hardwick C and Huber G (1973). Location of ambulatory care centers in a metropolitan area. Health Services Research 8(2): 121.

Smith H and Harper P (2010). Bicriteria efficiency/equity hierarchical location models. Technical report, University of Southampton.

Smith H, Laporte G and Harper P (2009). Locational analysis: Highlights of growth to maturity. Journal of the Operational Research Society 60(1): S140-S148.

Starfield B (1998). Primary Care: Balancing Health Needs, Services, and Technology. Oxford University Press: New York.

Verter V and Lapierre S (2002). Location of preventive health care facilities. Annals of Operations Research 110(1): 123-132.

WHO (1978). Declaration of Alma-Ata. International Conference on Primary Health Care, 6-12 September, Alma-Ata, http:// www.who.int/publications/almaata_declaration_en.pdf.

Zhang Y, Berman O and Verter V (2009). Incorporating congestion in preventive healthcare facility network design. European Journal of Operational Research 198(3): 922-935.

Received October 2011; accepted April 2012 after one revision 